\title{
Review
}

\section{Neuronal functions of FOXO/DAF-16}

\author{
Sun Y. Kim and Ashley E. Webb* \\ Department of Molecular Biology, Cell Biology and Biochemistry, Brown University, Providence, RI, USA
}

\begin{abstract}
The FOXO family of transcription factors plays a conserved role in longevity and tissue homeostasis across species. In the mammalian nervous system, emerging evidence has implicated FOXOs in cognitive performance, stem cell maintenance, regeneration, and protection against stress. Much of what we know about neuronal functions of FOXO emerged from recent studies in C. elegans. Similar to mammalian FOXO, the worm FOXO ortholog, called DAF-16, regulates learning and memory, regeneration, and stress resistance in neurons. Here, we discuss the current state of our knowledge of FOXO's functions in neurons in mammals and invertebrates, and highlight areas where our understanding is limited. Defining the function of FOXO factors in the healthy, aged, and diseased brain may have important implications for improving healthspan and treating neurodegenerative disease.
\end{abstract}

Keywords: FOXO, DAF-16, insulin/IGF signaling, brain, aging

\section{Introduction}

Cognitive decline is a major hallmark of aging, and is exacerbated in individuals suffering from neurodegenerative disease. The mechanisms of brain aging are complex and include alterations in connectivity, increased inflammation, decreased neurogenesis, damage by free radicals, and dysregulation of gene expression networks [1]. A number of signaling pathways have been implicated in maintaining normal cognitive function in the adult, including insulin/insulin-like growth factor (IGF) signaling. FOXO factors, known as DAF-16 in worms, are the key transcription factors downstream of insulin/IGF/AKT signaling (Fig. 1). FOXOs are expressed broadly across several tissue types, including the brain, and are critical effectors of cellular homeostasis, metabolism, and the stress response. Work first performed in invertebrates demonstrated that FOXOs regulate organismal longevity, and

\footnotetext{
*Corresponding author: Dr. Ashley Webb, Department of Molecular Biology, Cell Biology and Biochemistry, Brown University, Providence, RI 02903, USA. Tel.: +1 401863 6840; E-mail: Ashley_Webb@brown.edu.
}

increased FOXO activity can extend lifespan in various species [2]. Recent studies focused on the nervous system have implicated FOXOs in cognitive performance, regeneration, and neurodegenerative disease. In this review, we discuss the contribution of the FOXO family of transcription factors to neuronal function in invertebrates and mammals. We first examine the evidence implicating FOXO factors in learning and memory across species. We then discuss the known roles of these factors in regeneration of the nervous system. Finally, we consider the evidence implicating FOXOs in the response to stress, neurodegeneration, and normal brain aging.

\section{The FOXO family of transcription factors}

FOXO transcription factors function downstream of insulin/IGF signaling and play a conserved role in longevity and cellular homeostasis. FOXOs are members of the Forkhead family of transcription factors, which are distinguished by a conserved winged helix DNA binding domain known as the 'Forkhead box' [3]. C. elegans and Drosophila each have one 


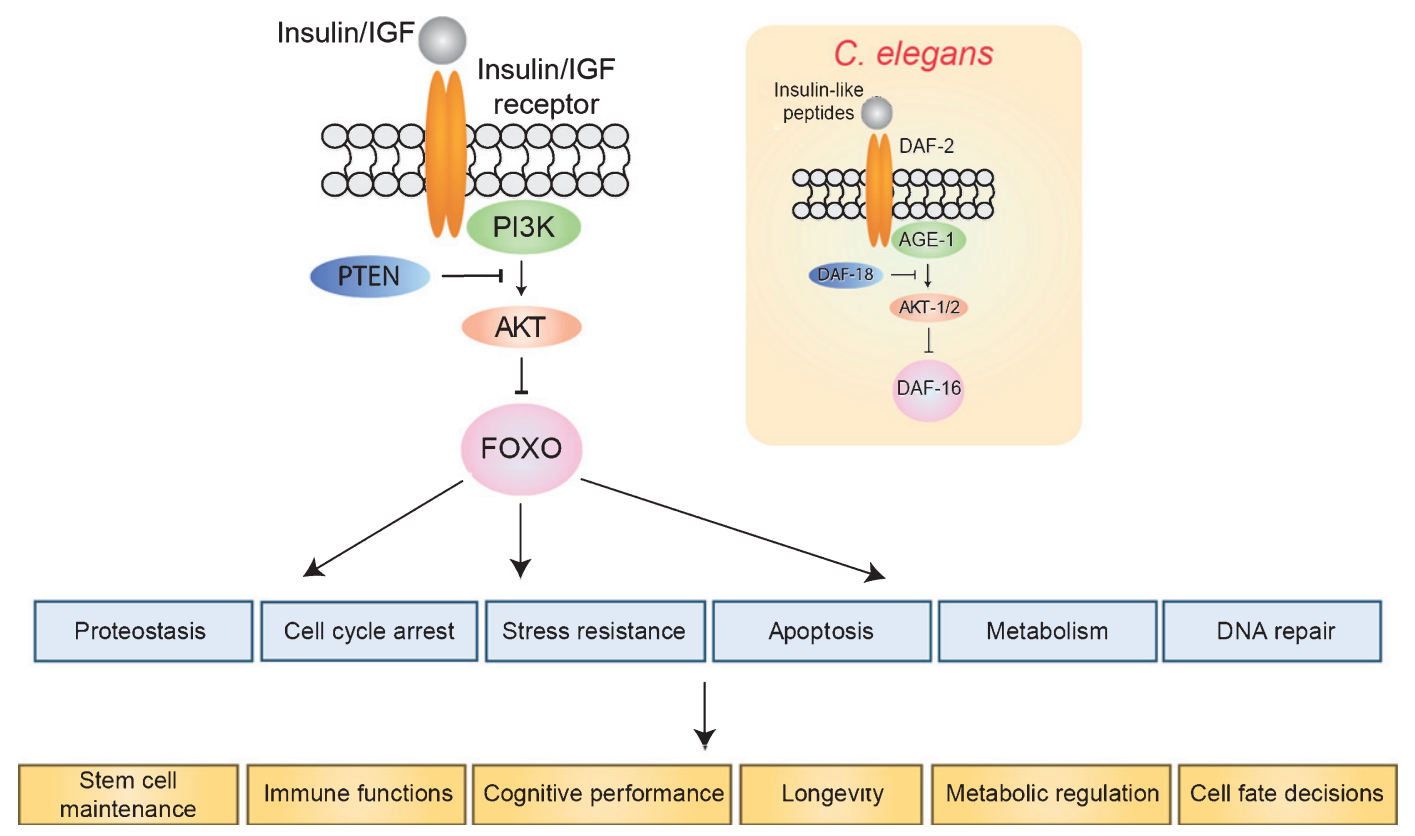

Fig. 1. The insulin/IGF signaling pathway. FOXO transcription factors are inhibited by insulin/IGF signaling. In the absence of insulin/IGF binding, FOXOs regulate a number of cellular processes (blue), which in turn affect tissue homeostasis and organismal longevity. Inset shows the orthologous pathway in C. elegans.

FOXO gene, called DAF-16 and dFOXO, respectively. Mammals have four different FOXO isoforms (FOXO1, 3, 4 and 6). When activated, FOXOs function as pioneer transcription factors and can remodel compacted chromatin, with or without the help of ATP-dependent chromatin remodelers $[4,5]$. FOXOs bind the consensus sequence TGTTTAC and primarily function as transcriptional activators, though repression has also been observed [6-8].

FOXOs are negatively regulated by insulin/IGF and other growth factor signals. In C. elegans, there is only one insulin/IGF receptor, known as DAF-2. Growth factor signaling activates the phosphoinositide 3-kinase (PI3K)-AKT pathway, and FOXOs possess three conserved AKT-dependent phosphorylation sites $[9,10]$. Phosphorylation at these sites inhibits FOXO activity by sequestering FOXO protein in the cytoplasm. Under conditions of low insulin or growth factors, FOXOs are unphosphorylated at the AKT-dependent phospho-sites, and are able to translocate into the nucleus and activate target genes. The FOXO6 isoform is an exception to this rule, as it does not shuttle between the nucleus and the cytoplasm in response to AKT, and is constitutively nuclear $[11,12]$. In addition to the insulin/IGF/AKT signaling axis, FOXOs receive inputs from a number of other factors and signaling pathways, depending on environmental conditions. For example, FOXOs are phosphorylated by AMPK in response to alterations in nutrient signaling [13], MST-1 and JNK under conditions of oxidative stress [14-16], and SGK under conditions that promote survival and cell cycle progression [17]. Additional covalent modifications to FOXO include methylation by SET9 and PRMT1, acetylation by p300 and CREB-binding protein, and deacetylation by SIRT1 [18-22]. The combination of these post-translational modifications has been proposed to function as a FOXO 'code', which directs context-specific gene expression programs [23]. Indeed, many mammalian targets of FOXO are tissue-specific, underscoring the complexity of FOXO's contribution to cell and tissue homeostasis [24].

\section{Contribution to cognitive function}

\subsection{Invertebrate studies}

C. elegans has emerged as a powerful model in neuroscience due to its relative simplicity, invariant cell lineage, stereotyped neuronal wiring, and behavioral plasticity. Each hermaphrodite worm has exactly 
A

\begin{tabular}{|c|c|c|}
\hline $\begin{array}{l}\text { Learning/memory } \\
\text { paradigm }\end{array}$ & $\begin{array}{l}\text { DAF-16's } \\
\text { function }\end{array}$ & $\begin{array}{l}\text { Primary neuronal } \\
\text { subtype involved }\end{array}$ \\
\hline Isothermal tracking & $\begin{array}{l}\text { Enhances } \\
\text { learning }\end{array}$ & AFD neurons \\
\hline Salt chemotaxis & $\begin{array}{l}\text { Inhibits } \\
\text { learning }\end{array}$ & ASER neuron \\
\hline $\begin{array}{l}\text { Olfactory association } \\
\text { (butanone) }\end{array}$ & $\begin{array}{l}\text { Promotes short } \\
\text { and long term } \\
\text { memory }\end{array}$ & AWC neurons \\
\hline
\end{tabular}

B
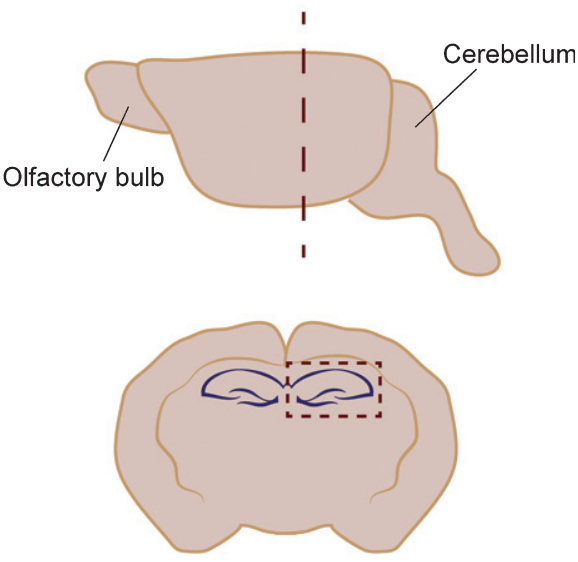

Rodent hippocampus

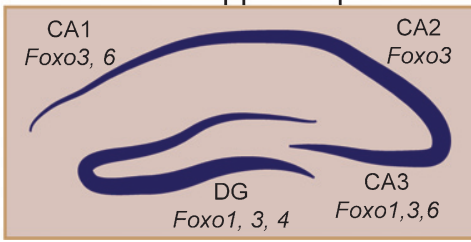

Fig. 2. FOXOs have been implicated in learning and memory in C. elegans and mice. (A) Summary of the evidence implicating DAF-16 in the regulation of learning and memory in C. elegans. (B) Schematic representation of the domains of the hippocampus that express different FOXO isoforms in the mouse. The upper panel depicts a side view of the rodent brain. A coronal section at the level of the hippocampus (dotted line in the side view) is shown in the center. The bottom image shows a zoomed-in schematic of the hippocampus, and indicates where the different mammalian FOXO isoforms are expressed.

302 neurons, and the connectivity of these cells is consistent between animals [25]. Thus, each individual neuron can be identified based on its unique position, connectivity, and morphology. In addition, worms respond to environmental stimuli and learn to modify their behavior in response to the particular stimulus. Learning and memory paradigms have been developed in C. elegans to assess associative learning, non-associative learning (habituation) and developmental imprinting [26]. The insulin signaling pathway has been implicated in associative learning, in which worms are able to learn and remember specific environmental cues, such as an odor, taste, or temperature. Behavioral studies, in combination with recently developed genomics approaches have revealed neuron-specific functions for DAF16/FOXO in learning and memory.

Murakami et al. observed that the insulin/IGF1 signaling pathway regulates associative learning using an isothermal tracking paradigm [27] (Fig. 2). In the isothermal tracking assay, animals learn to associate temperature with food, and performance in this assay decays with age. The authors found that age-1 (PI3K) and daf-2 mutants, which have increased DAF-16 activity, have a delayed age-associated decline in this assay. This delay is suppressed by daf-16 mutation. Thus, in this case, insulin/IGF signaling through the DAF-2 receptor reduces associative learning, whereas DAF-16 enhances it. In contrast, insulin/IGF-1 signaling has been reported to augment a different form of associative learning known as salt chemotaxis learning. This method uses salt starvation conditioning, in which the worms learn to associate salt with the absence of food. Learned salt aversion in this assay required insulin/IGF-1 signaling, as daf-2, age-1 (PI3K), and akt-1 mutants retained the ability to chemotax towards salt in naïve conditions, but were unable to develop a salt aversion [28, 29]. Conversely, daf-18/PTEN mutant worms, which have enhanced PI3K/AKT signaling, had increased associative learning in this assay compared to wild type animals. Why does insulin/IGF-1 signaling impair behavioral plasticity in one context (isothermal tracking) and promote it in another (salt avoidance behavior)? Interestingly, evidence suggests that the difference may be in the effectors downstream of AKT in each case. Whereas DAF-16 enhanced 
associative learning in isothermal tracking experiments, in the salt chemotaxis context, $d a f-16$ mutation only partially suppressed the learning defect of $d a f-$ 2 mutants. This finding indicates that other factors function downstream of the insulin/IGF-1 signaling axis in the salt starvation context. These signaling differences may reflect differences in the neuronal subtype involved, as the two types of learning are mediated by distinct sets of neurons. Consistent with this notion, Tomioka et al. show that the insulin/IGF1 signaling functions in a particular neuronal subtype (ASER neurons) in salt chemotaxis learning, whereas isothermal tracking is mediated primarily by AFD neurons $[28,30]$.

DAF-16 has also been shown to regulate memory performance during C. elegans aging. Kauffman et al. developed a positive olfactory association assay to assess learning and memory with age in worms. In this assay, worms learn to associate food with a specific odorant (butanone), for which chemosensation is mediated by the AWC neurons [31]. After the initial learning stages, worms are assessed for shortterm (2 hours) and long-term memory (16 hours). Long-term olfactory memory is the first neuronal function to be lost with age and, similar to mammals, this type of memory is regulated by CREB. Olfactory learning, chemotaxis and motility decline later in life (after one week of adulthood). In early adulthood, daf-2 mutants maintain short-term memory three times as long as wild type worms, and have enhanced long-term memory. Both functions are dependent on daf-16. However, with age, daf-2 mutant worms do not have an extension of longterm memory compared to wild type worms, and therefore do not maintain all forms of memory performance despite their increased longevity. This finding is consistent with the observation that insulin/IGF-1 signaling acts in the intestine, and not the neurons, to regulate longevity [32]. These studies raise the question of whether DAF-16 regulates a specific set of neuron-specific genes.

The identity of the neuronal DAF-16 targets remained elusive for a long time in part due to the challenge of performing tissue-specific gene expression profiling in C. elegans. Worms possess a tough outer cuticle that makes sample preparation difficult, and they cannot be manually dissected due to their small size. Only recently, Kaletsky et al. were able to define these neuronal-specific transcriptomes [33]. The authors used fluorescence activated cell sorting to isolate GFP-positive neurons from wild type, $d a f-2$ mutants and daf-2;daf-16 mutants, followed by
RNA-seq and comparison to whole worm transcriptomes. Consistent with neural-specific functions of DAF-16 that are separate from its role in longevity, neuron-specific DAF-16-regulated genes are distinct from longevity DAF-16 targets. Whole worm targets are most enriched in metabolism genes, whereas neuron targets are most enriched in neuronal functions (GPCR, ion channels, kinesins, etc.). Notably, regulation of these genes may be in part indirect since many of the putative DAF-16-regulated neuronal genes did not contain DAF-16 binding element sequence motifs. Nevertheless, the genes identified using this method are likely to be functional regulators of learning and memory directly or indirectly downstream of DAF16 since RNAi against $80 \%$ of the top targets resulted in associative memory deficits. Which of the neuronal targets are directly regulated by DAF- 16 remains to be determined. In addition, in future studies, as technology allows, it will be interesting to examine the expression networks downstream of DAF-16 in different neuronal subtypes since DAF-16 appears to be more important for maintaining youthful programs of gene expression in some neurons than others.

Together, these studies demonstrate that DAF-16 regulates a neuron-specific program of target genes whose function is to maintain learning and memory in the adult. In worms, neuronal DAF-16 targets are distinct from those regulating longevity. Similarly, dFOXO, the Drosophila FOXO ortholog, functions in the fat body, and not the brain to regulate lifespan in the fly [34].

\subsection{Mammalian studies}

The extensive evidence implicating the $C$. elegans FOXO ortholog in learning and memory raises the question of whether this function is conserved in other species. Moreover, insulin/IGF signaling has been strongly linked to cognition in mammals, and particular alleles of FOXO3 have been linked to enhanced cognitive function in humans with age [35]. However, the underlying mechanism remains unknown. All four FOXO isoforms are expressed in the mammalian brain (though FOXO4 is expressed at much lower levels than the others) [6, 36, 37]. Genetic mouse models have been generated for all FOXOs, and mice with complete knockout of Foxo3, 4 , and 6 are viable [38-40], whereas complete knockout of Foxol is embryonic lethal. Brain-targeted Foxol knockout mice (using a Nestin-Cre driver) have also been generated to circumvent the lethality 
of the complete knockout [41]. These mouse models have been used in several studies to investigate functions of FOXOs in the brain. However, thus far, studies investigating the contribution of mammalian FOXOs to learning and memory have been limited to FOXO6 [38]. Although they haven't been investigated in learning and memory functions, both FOXO1 and FOXO3 have been implicated in regulating anxiety-like behaviors in adult mice [41]. FOXO3 also functions in a reward center of the brain, known as the nucleus accumbens (ventral striatum), to modulate the behavioral response to cocaine [42]. These studies suggest functions for FOXO factors in serotonergic signaling and reward circuitry in the brain, respectively.

FOXO6 is the main FOXO isoform that has been linked to cognitive function in the mouse [38]. FOXO6 is strongly expressed in the CA1 and CA3 regions of the hippocampus, but not in the dentate gyrus (Fig. 2). Functionally, FOXO6 is required to establish neuronal polarity, and regulates a gene expression program in that is essential for neuronal connectivity in the hippocampus [43]. In the absence of FOXO6, associative learning in contextual fear conditioning and novel object recognition assays is normal, but mice are impaired in memory consolidation [38]. Precisely how FOXO6's function relates to that of other FOXOs in the brain remains unknown. FOXO3 is enriched in all regions of the hippocampus (dentate gyrus, CA1, CA2 and CA3), and FOXO1 is expressed in the dentate gyrus and in ventral CA3 pyramidal neurons (Fig. 2) [36]. Thus, based on gene expression patterns, FOXOs may have both overlapping and separate roles in the hippocampus. Functionally, the contribution of each factor remains unknown. Similar to FOXO1, 3 and 4, FOXO6 is negatively regulated by insulin/IGF-1 signaling. However, unlike the other isoforms, FOXO6 does not shuttle between the nucleus and the cytoplasm [11, 12], suggesting some differences in how this isoform is regulated. In addition, all four isoforms are highly similar in their DNA binding domains, but diverge outside of this region. Thus, the different FOXOs may partner with different cofactors to regulate different gene expression programs in the hippocampus, which regulate diverse cellular processes in learning and memory. Future studies, including behavioral analysis, expression profiling, and electrophysiological approaches will be necessary to address this important question.

In addition, it will be important to test whether a function of FOXOs in mammals is to preserve neuronal function with age, similar to their role in $C$. elegans. During mammalian aging, the coordination and integration of neural activity are disrupted, resulting in reduced cognitive capacity [1]. The underlying mechanisms, including the potential role of FOXO factors in cognitive aging, are likely to be complex. Intriguingly, expression profiling of the human brain during aging revealed dysregulation of synaptic genes with age [44]. Whether these are the same genes that are regulated by FOXO6 in the mouse hippocampus is unknown, but it suggests the possibility that FOXO6 may maintain memory performance with age by coordinating synaptic stability. However, paradoxically, insulin/IGF signaling is generally considered protective in the aging brain [45]. Insulin/IGF signaling supports neuronal survival, synaptogenesis, neurotransmission, neurogenesis, and memory retention. Conversely, knockout of the IGF-1 receptor or the Irs 2 signaling molecule are protective in Alzheimer's models [46-48]. Altogether, these findings suggest that cognitive performance depends on optimal levels of insulin/IGF signaling and FOXO activation in the brain. Additional studies will be required to fully understand how this balance is achieved, and define the role of the different FOXO family members in adult cognition.

\section{Axon growth and regeneration}

\subsection{Lessons from invertebrates}

Early studies employing electron microscopy in $C$. elegans suggested that neurons remain structurally intact in nematodes with age, despite deterioration of the surrounding tissue [49]. However, worms display reduced motility and associative learning with age [27], indicating that neurons are functionally impaired in aged animals. Indeed, more recent studies using fluorescent reporter strains have revealed defects in several neuronal subtypes with age, including mechanosensory neurons, GABAergic motor neurons, and cholinergic neurons. Here we consider the evidence suggesting that in different neuronal contexts, neurite outgrowth, structural maintenance, and regeneration are all regulated by DAF-16 during aging.

C. elegans aging is accompanied by several alterations to the nervous system, including increased dendrite branching, axonal defasciculation, neuronal blebbing, ectopic axonal outgrowth, and reduced 
regenerative ability. These features of neuronal aging are all regulated by DAF-16 and, interestingly, can be uncoupled from DAF-16's role in organismal aging. For example, mechanosensory and GABAeric neurons in aging worms develop ectopic branches, correlating with a decline in mobility and responsiveness to touch [50]. In addition to regulation by DAF-16, branching is also controlled by MAPK signaling involving the JNK-1 and JKK-1 kinases (but not other MAPK members). jnk- 1 and $j k k-1$ mutants have an accelerated branching of mechanosensory and GABAergic neurons, and have corresponding locomotory defects. Tissue-specific expression experiments showed that JNK-1 and DAF-16 function cell autonomously. However, unlike in the context of organismal longevity where JNK-1 acts directly upstream of DAF-16, in neurons these factors function independently to regulate branching. Interestingly, these experiments also uncoupled DAF-16's role in neurite branching from the rate of organismal aging since reducing daf-2 in all tissues but neurons extended lifespan, but did not promote more youthful branching morphologies [16].

DAF-16 also maintains the integrity of touch receptor neurons in C. elegans. During aging, these neurons develop blebs and aberrant branches, including ectopic axonal outgrowth from the soma [50-52]. Deterioration of synaptic integrity has also been observed with age in worms, which is reminiscent of what has been observed in humans [1, 52]. daf-2 mutants are delayed in the onset of these defects, and daf-16 is required for this phenotype. In contrast to daf-2 worms, eat-2 mutants do not exhibit delayed neuronal aging, indicating that not all long-lived mutants are protected from neuronal alterations with age. Therefore, simply delaying aging is not sufficient for neuroprotection, again suggesting that neuronal aging is regulated cell autonomously. Notably, some neurons in C. elegans do not have age-associated morphological abnormalities (e.g. inhibitory GABAergic motor neurons, nerve ring interneurons) [52]. What are the mechanisms that specifically act in some neuronal subtypes to maintain morphology and function with age? The mechanism has yet to be fully elucidated but two lines of evidence indicate that mitochondria may play a role. First, mitochondria are often associated with neurite branch sites [52]. And second, clk-1 mutants, which exhibit reduced respiration and extended lifespan, also have delayed neurite branching [50]. The precise effect that mitochondria, or the electron transport chain, have on neurite branching is not clear, but manipulating ROS levels using paraquat does not alter neurite branching, suggesting that dysfunctional branching is not caused by oxidative stress [50]. Future work will be required to define the precise mechanism downstream of DAF-16 in this process and whether regulation of mitochondrial function is involved.

C. elegans have the ability to regenerate neurons in adulthood, and this capacity is reduced with age. Adult neurons can be severed using laser axotomy, which allows for accurate and reproducible cutting of single neurons in vivo, followed by a stereotyped regenerative response [53]. This approach has been proposed as a model system to identify the molecular regulators of regeneration, and the mechanisms underlying its decline during aging $[54,55]$. Using this approach, Byrne et al. characterized the decay in GABA motor neuron regeneration with age [56]. Most young adult (day 1) animals were able to regenerate, but by day 5 of adulthood, only about one quarter of severed axons could initiate regeneration, and those that could initiate failed to fully extend toward their targets. The age-related decline in regenerative capacity was delayed in $d a f-2$ mutants, and this response required DAF-16. DAF-16 acts cell autonomously in neurons to regulate regeneration, independent of its functions in longevity. Moreover, other manipulations that extend lifespan (e.g. dietary restriction) do not improve regeneration, supporting the notion that improving regeneration is not simply a consequence of extending lifespan or healthspan. This result is somewhat surprising since DAF-16 has been found to be required for lifespan extension in response to some forms of dietary restriction. The mechanistic underpinnings responsible for this difference remains unknown, but one possibility is that it depends on the type of dietary restriction used. In the Byrne et al. study, eat-2 mutants were used to induce dietary restriction. However, DAF-16 is not completely necessary for eat-2-induced longevity $[57,58]$. Perhaps other methods of dietary restriction would enhance regeneration. Nevertheless, this study suggests that DAF-16 promotes neuronal regeneration via a cell-intrinsic mechanism independent of its role in longevity, as expression of DAF-16 specifically in neurons rescued the age-associated deterioration of motor neuron regeneration [56].

DAF-16 also regulates development of the nervous system, and this function may be conserved in mammals. During development, DAF-16 promotes neurite outgrowth in interneurons that modulate locomotory behavior and thermotaxis (AIY neurons) [59]. 
The morphology of these neurons is highly stereotyped during development, and disruptions in AIY growth can be easily visualized using fluorescence microscopy. daf-16 mutants are defective in AIY neurite outgrowth and, interestingly, this function of DAF-16 is performed by one particular DAF-16 isoform, DAF-16B. Previous studies had implicated the DAF-16A, D and F isoforms as the variants that regulate longevity [60], suggesting that the regulation of aging and neuronal patterning by DAF-16 can be uncoupled. Notably, the N-terminal half of the DNA binding domain is encoded by different exons in DAF-16A and B. Thus, differences in motif binding preferences and therefore target gene regulation may drive neuron-specific versus longevity functions of DAF-16. However, this has yet to be explored. DAF$16 \mathrm{~A}$ and $\mathrm{B}$ are also regulated by distinct promoters [61], which is also likely to contribute to their distinct functions. Importantly, Christensen et al. extend their findings to a mammalian system and find that FOXO is necessary for axon outgrowth in rat primary cerebellar granule neurons [59]. Simultaneous knockdown of FOXO1, 3, and 6 reduced axon length in these cells, and this phenotype could be rescued by expression of FOXO6. FOXO1 and FOXO3 were not tested, so the contribution of these isoforms to axon growth in mammals remains unclear. Nevertheless, these findings are consistent with the observation that FOXO6 knockout mice have decreased dendritic spine density in hippocampal neurons in vivo [38], and that FOXO6 regulates neuronal polarity.

\subsection{Axon regeneration in mammalian systems}

Unlike the central nervous system, the mammalian peripheral nervous system (PNS) possesses the ability to regenerate. Common types of PNS injury are lacerations, contusions and avulsion (detachment) type injuries. However, the rate of regeneration is slow (1-3 mm per day), and functional recovery is often incomplete. A number of genes associated with regeneration in mammals have been identified. These factors include neurotrophic factors and structural proteins that orchestrate the repair and regrowth of nerves. Induction of the regeneration program peaks at one week post injury, then declines over the course of 2-3 months [62]. Regeneration also depends on Schwann cells, which provide growth support to the regenerating axons. Similar to C. elegans, regeneration of peripheral nerves also declines with age in mammals [63, 64], and the PI3K-AKT signaling pathway has been implicated in the process of regeneration [62]. However, in contrast to what has been observed in the nematode, IGF-1 can stimulate regeneration of mammalian axons in response to injury [65]. Consistent with this observation, FOXO3 levels are reduced in neurons and glia within 48 hours of spinal cord injury, and gradually recover over the course of four weeks [66, 67]. This trajectory is anti-correlated with PCNA levels and expression of GAP-43, a presynaptic membrane protein that guides axon regeneration. The relative contribution of FOXO3 in neurons versus glia is not known. In a model of acute spinal cord contusion, FOXO3 was primarily downregulated in astrocytes, which are induced to proliferate in response to injury [67]. While these studies do not address the functional role of FOXO3 in astrocytes upon injury, these finding suggest that FOXO3 functions in some way to restrain scar formation after spinal cord injury.

\section{FOXOs and neurogenesis}

In mammalian embryonic and postnatal development, neural stem cells (NSCs) give rise to neurons and glia that shape the basic architecture of the central nervous system. The adult brain then retains some of its capacity to generate new functional neurons by preserving a small population of NSCs [68-71]. In the healthy brain, adult neurogenesis is restricted to the two neurogenic niches: the subventricular zone (SVZ) lining the lateral ventricles and the dentate gyrus (DG) region of the hippocampus. In these niche microenvironments, adult NSCs reside mostly in a quiescent state, but maintain their multipotency and ability to proliferate and self-renew [72]. Through integration of new neurons into the hippocampal and olfactory circuitry, adult neurogenesis allows mammals greater cognitive plasticity and contextual learning abilities [73]. However, adult neurogenesis also declines with age, and the subsequent failure to adequately replenish the brain with functional neurons may contribute to the cognitive decline of the aging brain [74-76]. In mammals, FOXOs are key regulators of stem cells, including NSCs. Complete knockout of Foxo3 or brain-specific triple knockout of Foxol, 3, and 4 results in a depletion of the NSC pool [77, 78]. In both cases, ablation of FOXO function significantly increases brain size, and induces precocious NSC proliferation during development and early adulthood. This initial surge in NSC 
proliferation in the absence of FOXOs is followed by a progressive decline in self-renewal potential, resulting in a premature depletion of the NSC reserve.

Genome-wide microarray analysis comparing Foxo3 knockout and wild type NSCs revealed that FOXO3 regulates expression of genes involved in quiescence, oxidative stress resistance, glucose metabolism and transport, and early neurogenesis [78]. Altogether, this program is critical for maintaining NSC functionality in the brain, and possibly during aging. For example, excess reactive oxygen species (ROS) contributes to functional decline in stem cells, and high levels of ROS can prematurely activate stem cells and deplete the reserve [79]. Extensive evidence has demonstrated that FOXOs protect various cell types, including stem cells, from ROS [80-82]. However, treatment of FOXOnull NSCs with $\mathrm{N}$-acetyl-cysteine to restore normal ROS levels only partially rescues NSC self-renewal [77], indicating that additional mechanisms are at play downstream of FOXOs in these cells. Interestingly, embryonic stem cell-derived neural progenitor cultures in a BMP4-induced quiescent state show enrichment for expression of FOXO-regulated genes [83, 84], suggesting a 'quiescence program' downstream of FOXOs. In contrast, FOXO-regulated genes in the NSCs do not overlap extensively with those in hematopoietic stem cells, indicating a neural-specific FOXO transcriptional network that is not shared by other stem cells [77].

ChIP-seq analysis of the direct targets of FOXO3 in adult NSCs has more precisely defined the mechanism by which FOXOs regulate NSCs [8]. FOXO3 binds over 2,000 direct targets in NSCs, and many of these targets are neural-specific (not bound by FOXOs in other mouse tissues) [24]. Intriguingly, neural-specific targets of FOXO include genes in the Alzheimer's disease presenilin pathway, though the functional relevance of these targets downstream of FOXOs has not been assessed. As expected, many of the target genes bound by FOXO3 in NSCs were also transcriptionally regulated by this factor based on transcriptome analysis [8]. However, a subset of FOXO3-bound genes were not observed to be transcriptionally regulated. The absence of regulation in these assays remains unknown, but may be due to redundancy among FOXO family members, or that these targets are only regulated under particular conditions. ChIP-seq analysis in NSCs also revealed a genome-wide network of targets shared with the pro-neural transcription factor ASCL1. FOXO3 and
ASCL1 are co-enriched at enhancers of Notch and Wnt signaling pathway genes known to regulate the balance between stemness and cell fate commitment [8]. Furthermore, FOXO3 inhibits ASCL1-dependent neurogenesis in progenitors as well as ASCL1's ability to directly reprogram fibroblasts into neurons. Altogether, these lines of evidence point to a role for FOXO in adult neurogenesis through maintenance of the NSC reserve.

In contrast to mammals, adult neurogenesis is absent in Drosophila, because no reserve of NSCs remains post-development. Interestingly, the single fly FOXO ortholog, dFOXO, plays a critical role in eliminating the neuroblasts in the young adult fly. As a result of reduced insulin-PI3KAKT signaling, dFOXO translocates into the nucleus of the neuroblasts and activates caspase-dependent apoptosis [85]. Absence of dFOXO and Reaper proapoptosis genes leads to long-term survival of the neuroblasts and prolonged neurogenesis in the adult mushroom body (center for learning and memory in Drosophila). Siegrist et al. demonstrated that these apoptosis-deficient neuroblasts have impaired growth, suggesting that dFOXO-mediated elimination of neuroblasts is beneficial to the fly. Therefore, while FOXOs regulate adult neural stem and progenitor cells in Drosophila and mice with different outcomes, their function as critical regulators of cellular homeostasis may optimize overall cognitive healthspan across species.

\section{Neuronal stress and neurodegenerative diseases}

Oxidative stress results from the overproduction of reactive oxygen species (ROS). ROS have been proposed to cause aging, and are generally considered to be detrimental to brain function, particularly in neurons. However, ROS also function as cellular messengers essential for cellular homeostasis. High levels of ROS generated in response to ischemic stroke or in neurodegeneration are a major cause of neuronal death. Across many cell types, FOXOs are activated in response to oxidative stress [21, 80, 86]. Once activated, FOXOs transcriptionally activate genes such as catalase and MnSOD, which in turn reduce intracellular ROS levels. Counterintuitively, insulin/IGF signaling protects against oxidative stress in the brain, including under conditions of extremely high oxidative stress, such as ischemic stroke. Insulin/IGF signaling is protective 
in the brain in part by blocking FOXO-induced neuronal death. Trophic deprivation and oxidative stress strongly activate FOXO in neurons. Under these conditions, FOXO induces pro-apoptotic genes such as BIM [87].

Activation of FOXO in conditions of oxidative stress involves several signaling molecules. Mammalian sterile 20-like kinase 1 (MST1) is a key regulator of oxidative stress-induced death in neurons and glia [14, 88]. In postnatal rat granule neurons and cortical astrocytes, MST1 is activated by oxidative stress and phosphorylates FOXO3 within its forkhead domain $[14,88]$. Phosphorylation at this site induces FOXO3 translocation to the nucleus and induction of cell death. Interestingly, this mechanism of FOXO activation is conserved across species, as $C$. elegans DAF-16 is phosphorylated by the worm ortholog of MST1 (CST-1) at a conserved serine residue. CST-1 overexpression extends lifespan in a daf-16 dependent manner. Davila et al. found that full activation of FOXO3 in response to oxidative stress in neurons involves inhibition of AKT, followed by activation of FOXO3 by JNK2 [89]. This mechanism appears to be specific to neurons, since p38/MAPK was not observed to inhibit AKT in response to $\mathrm{H}_{2} \mathrm{O}_{2}$ in astrocytes [90]. Consistent with these findings, FOXOs are strongly activated in in vivo rodent models of ischemic stroke, and may contribute to cell death in the infarct region [91, 92]. During normal aging, neuronal death is limited in the brain, suggesting that FOXO-induced neuronal death is not a major contributor to brain aging. However, in the context of neurodegenerative disease or in response to injury, oxidative stress can be an important contributor to neuronal death.

In addition to oxidative stress, FOXOs have emerged as important regulators of proteotoxic stress. Proteotoxicity is a major feature of neurodegenerative disease and occurs when cells accumulate damaged, misfolded, or aggregated proteins. A number of studies performed in C. elegans have shown that DAF-16 (or at least $d a f-2$ mutation) can promote clearance of $\mathrm{A} \beta$ [93] and polyglutamine [94-96], and alleviates the effects of mutant SOD1 [97,98]. Mechanistically, FOXOs promote autophagy, proteasome activity, and chaperone activity, and these activities are broadly conserved across species [99]. Nevertheless, investigation of the role FOXO in neurodegeneration or protein quality control in the mammalian brain has been limited so far. Most of the evidence associating FOXOs with proteostasis is from non-neuronal systems, although emerging evidence suggests FOXOs function similarly in neuronal cell types as well $[100,101]$. Moreover, studies directly linking FOXO with reduced proteotoxiciy in the diseased setting have been limited, although FOXO has been shown to clear $\alpha$-synuclein and defective mitochondria in dopaminergic neurons, which are affected in Parkinson's disease [102]. Together with evidence suggesting that reduced insulin/IGF signaling is protective in Alzheimer's disease [1, 46, 48], these findings suggest that FOXOs may have a broader role in protecting the brain from degeneration. Future studies will be required to address this important question.

\section{FOXO in the brain: An integrated model}

Studies performed in worms, flies and mice have implicated FOXO transcription factors in the regulation of NSCs, learning and memory, axon regeneration, and the neuronal stress response (Fig. 3). At first glance, these functions appear disparate and mechanistically disconnected. However, it must be considered that altered environmental conditions that affect cognitive performance such as aging, neurodegenerative disease, or metabolic syndromes (i.e. diabetes) would have global effects on FOXO activity. Thus, an integrated understanding of FOXO's many roles in the nervous system is necessary to fully grasp how the brain becomes

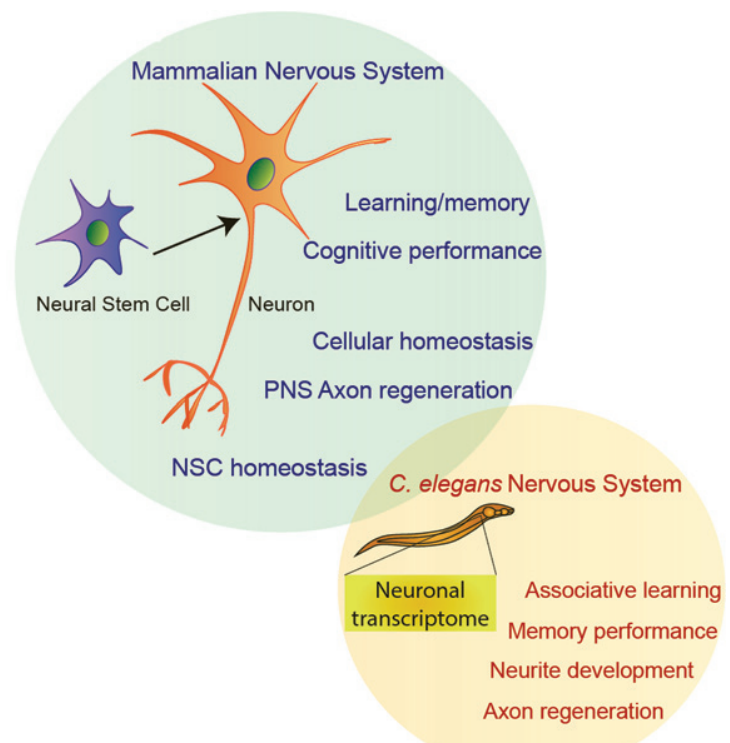

Fig. 3. The neuronal functions of FOXO transcription factors in C. elegans and mammals. 
dysfunctional in each of these circumstances. The key to building an integrated model of FOXO's role in the brain is in understanding the inputs that modulate its function and the outputs it produces in different contexts.

Many unanswered questions remain regarding the inputs regulating FOXOs in the brain. FOXOs receive inputs from the environment, including circulating and locally produced growth factors, metabolic cues, and oxidative stress signals. Do FOXOs elicit celltype specific responses to these different inputs in different neuronal subtypes and regions of the brain? Do these inputs change during aging or in neurodegenerative disease? Moreover, how are multiple signals incorporated to direct context and conditionspecific programs of gene expression? It remains possible that there may be additional upstream regulators of FOXOs in addition to the well-characterized PI3K-AKT signaling, MST, and JNK-mediated redox signaling. Identification of alternative regulators of FOXOs is likely to explain some of their cell type-specific actions. Another unresolved question regarding the inputs regulating FOXOs is the extent to which FOXO levels or activity are affected by interventions that extend lifespan. In the case of dietary restriction, DAF-16/FOXOs modulates the response in some, but not all, species, and the requirement also depends on the method of dietary restriction used [57, 58, 103-105]. Given the extensive evidence that FOXOs promote longevity, it is possible that they are activated in response to pharmacological interventions that delay aging, such as rapamycin, metformin, or 17- $\alpha$-estradiol, but this has yet to be fully investigated. Emerging evidence indicates that in addition to delayed organismal aging, interventions that extend lifespan also enhance cognitive health during aging. For example, lifelong treatment with rapamycin, an mTORC1 inhibitor, improves the agedependent decline in spatial memory in mice [106]. In addition, mTORC 1 activation induces neurogenesis and terminal differentiation in the adult mouse SVZ, suggesting a potential role of mTORC1 in the exhaustion of the NSC pool with age [107]. Metformin, which activates AMPK, has been shown to affect multiple stages of neurogenesis (proliferation, self-renewal, and differentiation) [108]. Whether FOXOs mediate these effects is unknown, but metformin can activate FOXO3 in stem-like glioma cells [109], suggesting a possible role for these factors in response to this drug. In summary, the extent to which FOXOs are involved in mediating the effects of longevity-promoting interventions and other inputs in the brain is likely to be an exciting area of future research.

Downstream of these inputs, how do FOXOs regulate a diverse range of processes? The answer is not fully understood, but emerging genomic evidence suggests that specific functions of FOXO factors rely on integration with other transcription factor networks. FOXOs interact with a number of transcription factors in different cell types and contexts (e.g. ETS, bHLH factors, b-catenin) [8, 110-112]. The functional diversity of the transcription factors that are associated with FOXOs may confer functional versatility, depending on the needs of the cell. For example, FOXO-associated factors can specifically direct a particular cellular response, such as autophagy or oxidative stress [99, 111]. In other contexts, FOXOs contribute to cell fate decisions by sharing common targets genomewide with cell type-specific transcriptional regulators $[8,112]$. These interactions with co-regulators can be synergistic or antagonistic. Recent work identified a number of new candidate co-regulators that may explain other aspects of FOXO's functions [24]. The precise interactions between FOXOs and other signaling pathways in the brain remain to be addressed. Given FOXO's role as one of the major nodes in the response to environmental cues, understanding how FOXOs are regulated in different contexts is likely to be key for understanding neuronal homeostasis in the healthy and diseased brain.

\section{Acknowledgments}

We thank Lauren Booth, Jana Lim, and Dervis Salih for critically reading the manuscript. This work was supported by an AFAR Research Grant for Junior Faculty to A.W., a Glenn/AFAR Graduate Student Scholarship to S.K., and NIH training grant AG041688.

\section{References}

[1] Bishop NA, Lu T, Yankner BA. Neural mechanisms of ageing and cognitive decline. Nature. 2010;464(7288):529-35.

[2] Kenyon CJ. The genetics of ageing. Nature. 2010; 464(7288):504-12.

[3] Kaestner KH, Knochel W, Martinez DE. Unified nomenclature for the winged helix/forkhead transcription factors. Genes Dev. 2000;14(2):142-6. 
[4] Zaret KS, Carroll JS. Pioneer transcription factors: Establishing competence for gene expression. Genes Dev. 2011;25(21):2227-41.

[5] Riedel CG, Dowen RH, Lourenco GF, Kirienko NV, Heimbucher T, West JA, et al. DAF-16 employs the chromatin remodeller SWI/SNF to promote stress resistance and longevity. Nat Cell Biol. 2013;15(5):491-501.

[6] Furuyama T, Nakazawa T, Nakano I, Mori N. Identification of the differential distribution patterns of mRNAs and consensus binding sequences for mouse DAF-16 homologues. Biochem J. 2000;349(Pt 2):629-34.

[7] Ramaswamy S, Nakamura N, Sansal I, Bergeron L, Sellers WR. A novel mechanism of gene regulation and tumor suppression by the transcription factor FKHR. Cancer Cell. 2002;2(1):81-91.

[8] Webb AE, Pollina EA, Vierbuchen T, Urban N, Ucar D, Leeman DS, et al. FOXO3 shares common targets with ASCL1 genome-wide and inhibits ASCL1-dependent neurogenesis. Cell Rep. 2013;4(3):477-91.

[9] Brunet A, Bonni A, Zigmond MJ, Lin MZ, Juo P, Hu LS, et al. Akt promotes cell survival by phosphorylating and inhibiting a Forkhead transcription factor. Cell. 1999;96(6):857-68.

[10] Kops GJ, de Ruiter ND, De Vries-Smits AM, Powell DR, Bos JL, Burgering BM. Direct control of the Forkhead transcription factor AFX by protein kinase B. Nature. 1999;398(6728):630-4.

[11] Jacobs FM, van der Heide LP, Wijchers PJ, Burbach JP, Hoekman MF, Smidt MP. FoxO6, a novel member of the FoxO class of transcription factors with distinct shuttling dynamics. J Biol Chem. 2003;278(38):35959-67.

[12] van der Heide LP, Jacobs FM, Burbach JP, Hoekman MF, Smidt MP. FoxO6 transcriptional activity is regulated by Thr26 and Ser184, independent of nucleo-cytoplasmic shuttling. Biochem J. 2005;391(Pt 3):623-9.

[13] Greer EL, Oskoui PR, Banko MR, Maniar JM, Gygi MP, Gygi SP, et al. The energy sensor AMP-activated protein kinase directly regulates the mammalian $\mathrm{FOXO} 3$ transcription factor. J Biol Chem. 2007;282(41):30107-19.

[14] Lehtinen MK, Yuan Z, Boag PR, Yang Y, Villen J, Becker $\mathrm{EB}$, et al. A conserved MST-FOXO signaling pathway mediates oxidative-stress responses and extends life span. Cell. 2006;125(5):987-1001.

[15] Essers MA, Weijzen S, de Vries-Smits AM, Saarloos I, de Ruiter ND, Bos JL, et al. FOXO transcription factor activation by oxidative stress mediated by the small GTPase Ral and JNK. EMBO J. 2004;23(24):4802-12.

[16] Oh SW, Mukhopadhyay A, Svrzikapa N, Jiang F, Davis RJ, Tissenbaum HA. JNK regulates lifespan in Caenorhabditis elegans by modulating nuclear translocation of forkhead transcription factor/DAF-16. Proc Natl Acad Sci U S A. 2005;102(12):4494-9.

[17] Brunet A, Park J, Tran H, Hu LS, Hemmings BA, Greenberg ME. Protein kinase SGK mediates survival signals by phosphorylating the forkhead transcription factor FKHRL1 (FOXO3a). Mol Cell Biol. 2001;21(3):952-65.

[18] Calnan DR, Webb AE, White JL, Stowe TR, Goswami $\mathrm{T}$, Shi X, et al. Methylation by Set 9 modulates FoxO3 stability and transcriptional activity. Aging (Albany NY). 2012;4(7):462-79.

[19] Yamagata K, Daitoku H, Takahashi Y, Namiki K, Hisatake $\mathrm{K}$, Kako K, et al. Arginine methylation of FOXO transcrip- tion factors inhibits their phosphorylation by Akt. Mol Cell. 2008;32(2):221-31.

[20] Mahmud DL, M GA, Deb DK, Platanias LC, Uddin S, Wickrema A. Phosphorylation of forkhead transcription factors by erythropoietin and stem cell factor prevents acetylation and their interaction with coactivator p300 in erythroid progenitor cells. Oncogene. 2002;21(10):155662.

[21] Brunet A, Sweeney LB, Sturgill JF, Chua KF, Greer $\mathrm{PL}$, Lin Y, et al. Stress-dependent regulation of FOXO transcription factors by the SIRT1 deacetylase. Science. 2004;303(5666):2011-5.

[22] van der Horst A, Tertoolen LG, de Vries-Smits LM, Frye RA, Medema RH, Burgering BM. FOXO4 is acetylated upon peroxide stress and deacetylated by the longevity protein hSir2(SIRT1). J Biol Chem. 2004;279(28):28873-9.

[23] Calnan DR, Brunet A. The FoxO code. Oncogene. 2008;27(16):2276-88.

[24] Webb AE, Kundaje A, Brunet A. Characterization of the direct targets of FOXO transcription factors throughout evolution. Aging Cell. 2016;15(4):673-85.

[25] White JG, Southgate E, Thomson JN, Brenner S. The structure of the nervous system of the nematode Caenorhabditis elegans. Philos Trans R Soc Lond B Biol Sci. 1986;314(1165):1-340.

[26] Ardiel EL, Rankin CH. An elegant mind: Learning and memory in Caenorhabditis elegans. Learn Mem. 2010;17(4):191-201.

[27] Murakami H, Bessinger K, Hellmann J, Murakami S. Aging-dependent and -independent modulation of associative learning behavior by insulin/insulin-like growth factor-1 signal in Caenorhabditis elegans. J Neurosci. 2005;25(47): 10894-904.

[28] Tomioka M, Adachi T, Suzuki H, Kunitomo H, Schafer WR, Iino Y. The insulin/PI 3-kinase pathway regulates salt chemotaxis learning in Caenorhabditis elegans. Neuron. 2006;51(5):613-25.

[29] Vellai T, McCulloch D, Gems D, Kovacs AL. Effects of sex and insulin/insulin-like growth factor-1 signaling on performance in an associative learning paradigm in Caenorhabditis elegans. Genetics. 2006;174(1):309-16.

[30] Garrity PA, Goodman MB, Samuel AD, Sengupta P. Running hot and cold: Behavioral strategies, neural circuits, and the molecular machinery for thermotaxis in C. elegans and Drosophila. Genes Dev. 2010;24(21):2365-82.

[31] Kauffman AL, Ashraf JM, Corces-Zimmerman MR, Landis $\mathrm{JN}$, Murphy CT. Insulin signaling and dietary restriction differentially influence the decline of learning and memory with age. PLoS Biol. 2010;8(5):e1000372.

[32] Libina N, Berman JR, Kenyon C. Tissue-specific activities of C. elegans DAF-16 in the regulation of lifespan. Cell. 2003;115(4):489-502.

[33] Kaletsky R, Lakhina V, Arey R, Williams A, Landis J, Ashraf J, et al. The C. elegans adult neuronal IIS/FOXO transcriptome reveals adult phenotype regulators. Nature. 2016;529(7584):92-6.

[34] Hwangbo DS, Gershman B, Tu MP, Palmer M, Tatar M. Drosophila dFOXO controls lifespan and regulates insulin signalling in brain and fat body. Nature. 2004;429(6991):562-6.

[35] Willcox BJ, Donlon TA, He Q, Chen R, Grove JS, Yano K, et al. FOXO3A genotype is strongly associated with human 
longevity. Proc Natl Acad Sci U S A. 2008;105(37):1398792.

[36] Hoekman MF, Jacobs FM, Smidt MP, Burbach JP. Spatial and temporal expression of FoxO transcription factors in the developing and adult murine brain. Gene Expr Patterns. 2006;6(2):134-40.

[37] Biggs WH, Cavenee WK, Arden KC. Identification and characterization of members of the FKHR (FOX O) subclass of winged-helix transcription factors in the mouse. Mamm Genome. 2001;12(6):416-25.

[38] Salih DA, Rashid AJ, Colas D, de la Torre-Ubieta L, Zhu RP, Morgan AA, et al. FoxO6 regulates memory consolidation and synaptic function. Genes Dev. 2012;26(24): 2780-801.

[39] Castrillon DH, Miao L, Kollipara R, Horner JW, DePinho RA. Suppression of ovarian follicle activation in mice by the transcription factor Foxo3a. Science. 2003;301(5630): 215-8.

[40] Hosaka T, Biggs WH, Tieu D, Boyer AD, Varki NM, Cavenee WK, et al. Disruption of forkhead transcription factor (FOXO) family members in mice reveals their functional diversification. Proc Natl Acad Sci U S A. 2004;101(9):2975-80.

[41] Polter A, Yang S, Zmijewska AA, van Groen T, Paik JH, Depinho RA, et al. Forkhead box, class $\mathrm{O}$ transcription factors in brain: Regulation and behavioral manifestation. Biol Psychiatry. 2009;65(2):150-9.

[42] Ferguson D, Shao N, Heller E, Feng J, Neve R, Kim $\mathrm{HD}$, et al. SIRT1-FOXO3a regulate cocaine actions in the nucleus accumbens. J Neurosci. 2015;35(7):3100-11.

[43] de la Torre-Ubieta L, Gaudilliere B, Yang Y, Ikeuchi Y, Yamada T, DiBacco S, et al. A FOXO-Pak1 transcriptional pathway controls neuronal polarity. Genes Dev. 2010;24(8): 799-813.

[44] Lu T, Pan Y, Kao SY, Li C, Kohane I, Chan J, et al. Gene regulation and DNA damage in the ageing human brain. Nature. 2004;429(6994):883-91.

[45] Fernandez AM, Torres-Aleman I. The many faces of insulin-like peptide signalling in the brain. Nat Rev Neurosci. 2012;13(4):225-39.

[46] Cohen E, Paulsson JF, Blinder P, Burstyn-Cohen T, Du D, Estepa G, et al. Reduced IGF-1 signaling delays age-associated proteotoxicity in mice. Cell. 2009;139(6): 1157-69.

[47] Freude S, Hettich MM, Schumann C, Stohr O, Koch L, Kohler C, et al. Neuronal IGF-1 resistance reduces Abeta accumulation and protects against premature death in a model of Alzheimer's disease. FASEB J. 2009;23(10): 3315-24.

[48] Killick R, Scales G, Leroy K, Causevic M, Hooper C, Irvine EE, et al. Deletion of Irs2 reduces amyloid deposition and rescues behavioural deficits in APP transgenic mice. Biochem Biophys Res Commun. 2009;386(1):257-62.

[49] Herndon LA, Schmeissner PJ, Dudaronek JM, Brown PA, Listner KM, Sakano Y, et al. Stochastic and genetic factors influence tissue-specific decline in ageing C. elegans. Nature. 2002;419(6909):808-14.

[50] Tank EM, Rodgers KE, Kenyon C. Spontaneous age-related neurite branching in Caenorhabditis elegans. J Neurosci. 2011;31(25):9279-88.

[51] Pan CL, Peng CY, Chen CH, McIntire S. Genetic analysis of age-dependent defects of the Caenorhabditis elegans touch receptor neurons. Proc Natl Acad Sci U S A. 2011;108(22):9274-9.

[52] Toth ML, Melentijevic I, Shah L, Bhatia A, Lu K, Talwar A, et al. Neurite sprouting and synapse deterioration in the aging Caenorhabditis elegans nervous system. J Neurosci. 2012;32(26):8778-90.

[53] Yanik MF, Cinar H, Cinar HN, Chisholm AD, Jin Y, BenYakar A. Neurosurgery: Functional regeneration after laser axotomy. Nature. 2004;432(7019):822.

[54] Byrne AB, Edwards TJ, Hammarlund M. In vivo laser axotomy in C. elegans. J Vis Exp. 2011(51).

[55] Nix P, Hammarlund M, Hauth L, Lachnit M, Jorgensen EM, Bastiani M. Axon regeneration genes identified by RNAi screening in C. elegans. J Neurosci. 2014;34(2):629-45.

[56] Byrne AB, Walradt T, Gardner KE, Hubbert A, Reinke V, Hammarlund M. Insulin/IGF1 signaling inhibits age-dependent axon regeneration. Neuron. 2014;81(3): 561-73.

[57] Greer EL, Brunet A. Different dietary restriction regimens extend lifespan by both independent and overlapping genetic pathways in C. elegans. Aging Cell. 2009;8(2):11327.

[58] Lakowski B, Hekimi S. The genetics of caloric restriction in Caenorhabditis elegans. Proc Natl Acad Sci U S A. 1998; 95(22):13091-6.

[59] Christensen R, de la Torre-Ubieta L, Bonni A, ColonRamos DA. A conserved PTEN/FOXO pathway regulates neuronal morphology during C. elegans development. Development. 2011;138(23):5257-67.

[60] Kwon ES, Narasimhan SD, Yen K, Tissenbaum HA. A new DAF-16 isoform regulates longevity. Nature. 2010;466(7305):498-502.

[61] Lee RY, Hench J, Ruvkun G. Regulation of C. elegans DAF16 and its human ortholog FKHRL1 by the daf- 2 insulinlike signaling pathway. Curr Biol. 2001;11(24):1950-7.

[62] Krishnan A, Duraikannu A, Zochodne DW. Releasing brakes to nerve regeneration: Intrinsic molecular targets. Eur J Neurosci. 2016;43(3):297-308.

[63] Pestronk A, Drachman DB, Griffin JW. Effects of aging on nerve sprouting and regeneration. Exp Neurol. 1980;70(1):65-82.

[64] Verdu E, Buti M, Navarro X. The effect of aging on efferent nerve fibers regeneration in mice. Brain Res. 1995;696(12):76-82.

[65] Kanje M, Skottner A, Sjoberg J, Lundborg G. Insulin-like growth factor I (IGF-I) stimulates regeneration of the rat sciatic nerve. Brain Res. 1989;486(2):396-8.

[66] Wang Y, Liu Y, Chen Y, Shi S, Qin J, Xiao F, et al. Peripheral nerve injury induces down-regulation of Foxo3a and p27kip1 in rat dorsal root ganglia. Neurochem Res. 2009;34(5):891-8.

[67] Zhang S, Huan W, Wei H, Shi J, Fan J, Zhao J, et al. FOXO3a/p27kip1 expression and essential role after acute spinal cord injury in adult rat. J Cell Biochem. 2013;114(2): 354-65.

[68] Kempermann G, Kuhn HG, Gage FH. Experience-induced neurogenesis in the senescent dentate gyrus. J Neurosci. 1998;18(9):3206-12 .

[69] Doetsch F, Garcia-Verdugo JM, Alvarez-Buylla A. Cellular composition and three-dimensional organization of the subventricular germinal zone in the adult mammalian brain. J Neurosci. 1997;17(13):5046-61. 
[70] Lois C, Alvarez-Buylla A. Proliferating subventricular zone cells in the adult mammalian forebrain can differentiate into neurons and glia. Proc Natl Acad Sci U S A. 1993;90(5):2074-7.

[71] Altman J, Bayer SA. Migration and distribution of two populations of hippocampal granule cell precursors during the perinatal and postnatal periods. J Comp Neurol. 1990;301(3):365-81.

[72] Doetsch F, Caille I, Lim DA, Garcia-Verdugo JM, AlvarezBuylla A. Subventricular zone astrocytes are neural stem cells in the adult mammalian brain. Cell. 1999;97(6): 703-16.

[73] Urban N, Guillemot F. Neurogenesis in the embryonic and adult brain: Same regulators, different roles. Front Cell Neurosci. 2014;8:396.

[74] Enwere E, Shingo T, Gregg C, Fujikawa H, Ohta S, Weiss S. Aging results in reduced epidermal growth factor receptor signaling, diminished olfactory neurogenesis, and deficits in fine olfactory discrimination. J Neurosci. 2004;24(38):8354-65.

[75] Lugert S, Basak O, Knuckles P, Haussler U, Fabel K, Gotz $\mathrm{M}$, et al. Quiescent and active hippocampal neural stem cells with distinct morphologies respond selectively to physiological and pathological stimuli and aging. Cell Stem Cell. 2010;6(5):445-56.

[76] Tropepe V, Craig CG, Morshead CM, van der Kooy D. Transforming growth factor-alpha null and senescent mice show decreased neural progenitor cell proliferation in the forebrain subependyma. J Neurosci. 1997;17(20):7850-9.

[77] Paik JH, Ding Z, Narurkar R, Ramkissoon S, Muller F, Kamoun WS, et al. FoxOs cooperatively regulate diverse pathways governing neural stem cell homeostasis. Cell Stem Cell. 2009;5(5):540-53.

[78] Renault VM, Rafalski VA, Morgan AA, Salih DA, Brett JO, Webb AE, et al. FoxO3 regulates neural stem cell homeostasis. Cell Stem Cell. 2009;5(5):527-39.

[79] Rossi DJ, Jamieson CH, Weissman IL. Stems cells and the pathways to aging and cancer. Cell. 2008;132(4):681-96.

[80] Kops GJ, Dansen TB, Polderman PE, Saarloos I, Wirtz KW, Coffer PJ, et al. Forkhead transcription factor FOXO3a protects quiescent cells from oxidative stress. Nature. 2002;419(6904):316-21.

[81] Tothova Z, Kollipara R, Huntly BJ, Lee BH, Castrillon DH, Cullen DE, et al. FoxOs are critical mediators of hematopoietic stem cell resistance to physiologic oxidative stress. Cell. 2007;128(2):325-39.

[82] Yeo H, Lyssiotis CA, Zhang Y, Ying H, Asara JM, Cantley LC, et al. FoxO3 coordinates metabolic pathways to maintain redox balance in neural stem cells. EMBO J. 2013;32(19):2589-602.

[83] Mira H, Andreu Z, Suh H, Lie DC, Jessberger S, Consiglio A, et al. Signaling through BMPR-IA regulates quiescence and long-term activity of neural stem cells in the adult hippocampus. Cell Stem Cell. 2010;7(1):78-89.

[84] Martynoga B, Mateo JL, Zhou B, Andersen J, Achimastou A, Urban N, et al. Epigenomic enhancer annotation reveals a key role for NFIX in neural stem cell quiescence. Genes Dev. 2013;27(16):1769-86.

[85] Siegrist SE, Haque NS, Chen CH, Hay BA, Hariharan IK. Inactivation of both Foxo and reaper promotes long-term adult neurogenesis in Drosophila. Curr Biol. 2010;20(7):643-8.
[86] Nemoto S, Finkel T. Redox regulation of forkhead proteins through a p66shc-dependent signaling pathway. Science. 2002;295(5564):2450-2.

[87] Gilley J, Coffer PJ, Ham J. FOXO transcription factors directly activate bim gene expression and promote apoptosis in sympathetic neurons. J Cell Biol. 2003;162(4):613-22.

[88] Lee SJ, Seo BR, Choi EJ, Koh JY. The role of reciprocal activation of cAbl and Mst1 in the oxidative death of cultured astrocytes. Glia. 2014;62(4):639-48.

[89] Davila D, Torres-Aleman I. Neuronal death by oxidative stress involves activation of FOXO3 through a twoarm pathway that activates stress kinases and attenuates insulin-like growth factor I signaling. Mol Biol Cell. 2008;19(5):2014-25.

[90] Davila D, Fernandez S, Torres-Aleman I. Astrocyte Resilience to Oxidative Stress Induced by Insulin-like Growth Factor I (IGF-I) Involves Preserved AKT (Protein Kinase B) Activity. J Biol Chem. 2016;291(5):2510-23.

[91] Kawano T, Morioka M, Yano S, Hamada J, Ushio Y, Miyamoto E, et al. Decreased akt activity is associated with activation of forkhead transcription factor after transient forebrain ischemia in gerbil hippocampus. J Cereb Blood Flow Metab. 2002;22(8):926-34.

[92] Shioda N, Ishigami T, Han F, Moriguchi S, Shibuya M, Iwabuchi Y, et al. Activation of phosphatidylinositol 3kinase/protein kinase B pathway by a vanadyl compound mediates its neuroprotective effect in mouse brain ischemia. Neuroscience. 2007;148(1):221-9.

[93] Cohen E, Bieschke J, Perciavalle RM, Kelly JW, Dillin A. Opposing activities protect against age-onset proteotoxicity. Science. 2006;313(5793):1604-10.

[94] Hsu AL, Murphy CT, Kenyon C. Regulation of aging and age-related disease by DAF-16 and heat-shock factor. Science. 2003;300(5622):1142-5.

[95] Morley JF, Brignull HR, Weyers JJ, Morimoto RI. The threshold for polyglutamine-expansion protein aggregation and cellular toxicity is dynamic and influenced by aging in Caenorhabditis elegans. Proc Natl Acad Sci U S A. 2002;99(16):10417-22.

[96] Parker JA, Arango M, Abderrahmane S, Lambert E, Tourette C, Catoire H, et al. Resveratrol rescues mutant polyglutamine cytotoxicity in nematode and mammalian neurons. Nat Genet. 2005;37(4):349-50.

[97] Boccitto M, Lamitina T, Kalb RG. Daf-2 signaling modifies mutant SOD1 toxicity in C. elegans. PLoS One. 2012;7(3):e33494.

[98] Mojsilovic-Petrovic J, Nedelsky N, Boccitto M, Mano I, Georgiades SN, Zhou W, et al. FOXO3a is broadly neuroprotective in vitro and in vivo against insults implicated in motor neuron diseases. J Neurosci. 2009;29(25):823647.

[99] Webb AE, Brunet A. FOXO transcription factors: Key regulators of cellular quality control. Trends Biochem Sci. 2014;39(4):159-69.

[100] Xu P, Das M, Reilly J, Davis RJ. JNK regulates FoxO-dependent autophagy in neurons. Genes Dev. 2011;25(4):310-22.

[101] Vilchez D, Boyer L, Morantte I, Lutz M, Merkwirth C, Joyce D, et al. Increased proteasome activity in human embryonic stem cells is regulated by PSMD11. Nature. 2012;489(7415):304-8. 
[102] Pino E, Amamoto R, Zheng L, Cacquevel M, Sarria JC, Knott GW, et al. FOXO3 determines the accumulation of alpha-synuclein and controls the fate of dopaminergic neurons in the substantia nigra. Hum Mol Genet. 2014;23(6):1435-52.

[103] Shimokawa I, Komatsu T, Hayashi N, Kim SE, Kawata T, Park S, et al. The life-extending effect of dietary restriction requires Foxo3 in mice. Aging Cell. 2015;14(4):707-9.

[104] Min KJ, Yamamoto R, Buch S, Pankratz M, Tatar M. Drosophila lifespan control by dietary restriction independent of insulin-like signaling. Aging Cell. 2008;7(2):199-206.

[105] Giannakou ME, Goss M, Partridge L. Role of dFOXO in lifespan extension by dietary restriction in Drosophila melanogaster: Not required, but its activity modulates the response. Aging Cell. 2008;7(2):187-98.

[106] Majumder S, Caccamo A, Medina DX, Benavides AD, Javors MA, Kraig E, et al. Lifelong rapamycin administration ameliorates age-dependent cognitive deficits by reducing IL-1beta and enhancing NMDA signaling. Aging Cell. 2012;11(2):326-35.

[107] Mahoney C, Feliciano DM, Bordey A, Hartman NW. Switching on mTORC1 induces neurogenesis but not proliferation in neural stem cells of young mice. Neurosci Lett. 2016;614:112-8.
[108] Fatt M, Hsu K, He L, Wondisford F, Miller FD, Kaplan DR, et al. Metformin Acts on Two Different Molecular Pathways to Enhance Adult Neural Precursor Proliferation/Self-Renewal and Differentiation. Stem Cell Reports. 2015;5(6):988-95.

[109] Sato A, Sunayama J, Okada M, Watanabe E, Seino S, Shibuya K, et al. Glioma-initiating cell elimination by metformin activation of FOXO3 via AMPK. Stem Cells Transl Med. 2012;1(11):811-24.

[110] Alic N, Giannakou ME, Papatheodorou I, Hoddinott MP, Andrews TD, Bolukbasi E, et al. Interplay of dFOXO and two ETS-family transcription factors determines lifespan in Drosophila melanogaster. PLoS Genet. 2014;10(9):e1004619.

[111] Essers MA, de Vries-Smits LM, Barker N, Polderman $\mathrm{PE}$, Burgering BM, Korswagen HC. Functional interaction between beta-catenin and FOXO in oxidative stress signaling. Science. 2005;308(5725):1181-4.

[112] Lin YC, Jhunjhunwala S, Benner C, Heinz S, Welinder E, Mansson R, et al. A global network of transcription factors, involving E2A, EBF1 and Foxo1, that orchestrates B cell fate. Nat Immunol. 2010;11(7):635-43. 